\title{
High-Flow Nasal Cannula versus Noninvasive Positive Pressure Ventilation in Patients with Heart Failure after Extubation: An Observational Cohort Study
}

\author{
Che-Jung Chang $\mathbb{D}^{1,2}$ Ling-Ling Chiang $\mathbb{D}^{2,3}$ Kuan-Yuan Chen, ${ }^{2}$ Po-Hao Feng, ${ }^{2,4}$ \\ Chien-Ling Su $\mathbb{1},{ }^{2,3}$ and Han-Shui Hsu $\mathbb{1}^{1}$ \\ ${ }^{1}$ Institute of Emergency and Critical Care Medicine, National Yang-Ming University, Taipei, Taiwan \\ ${ }^{2}$ Division of Pulmonary Medicine, Department of Internal Medicine, Shuang Ho Hospital, Taipei Medical University, \\ Taipei, Taiwan \\ ${ }^{3}$ School of Respiratory Therapy, College of Medicine, Taipei Medical University, Taipei, Taiwan \\ ${ }^{4}$ Division of Pulmonary Medicine, Department of Internal Medicine, School of Medicine, College of Medicine, \\ Taipei Medical University, Taipei, Taiwan
}

Correspondence should be addressed to Chien-Ling Su; 10038@s.tmu.edu.tw and Han-Shui Hsu; hsuhs@vghtpe.gov.tw

Received 22 February 2020; Revised 24 April 2020; Accepted 6 May 2020; Published 3 July 2020

Academic Editor: Inmaculada Alfageme

Copyright (c) 2020 Che-Jung Chang et al. This is an open access article distributed under the Creative Commons Attribution License, which permits unrestricted use, distribution, and reproduction in any medium, provided the original work is properly cited.

\begin{abstract}
Noninvasive positive pressure ventilation (NPPV) has been widely applied in patients with high-risk extubation failure, including heart failure. High-flow nasal cannula (HFNC) has been demonstrated to benefit patients with heart failure by reducing cardiac preload. This study aimed to compare the effectiveness of HFNC to NPPV for preventing extubation failure in patients with heart failure. This 3-year retrospective and single-center cohort study included patients with heart failure with left ventricular ejection fraction $<50 \%$ who received prophylactic HFNC or NPPV after scheduled extubation from January 2015 to January 2018 from a medical center with four adult intensive care units. Demographics, comorbidities, diagnosis, and weaning status were collected. The primary outcome was treatment failure within 72 hours after extubation, which was defined as escalation to NPPV or reintubation in the HFNC group and was defined as requiring reintubation in the NPPV group. Secondary outcomes were reintubation within 72 hours, reintubation, duration of stay, and mortality during the intensive care unit and hospital stay. Of the 104 patients analyzed, characteristics of 58 patients in the HFNC group and 46 patients in the NPPV group were compared. The treatment failure within 72 hours in the two groups was not significantly different $(25.9 \%$ vs $13 \%, p=0.106)$. Hypoxemic respiratory failure related treatment failure was significantly higher in the HFNC group. Prophylactic HFNC as first-line therapy had a comparable rate of reintubation within 72 hours to the prophylactic NPPV alone $(17.2 \%$ vs $13 \%, p=0.556)$. Other secondary outcomes were similar between the two groups. Among patients with heart failure, HFNC was not inferior to NPPV for preventing extubation failure and reintubation. However, in case of an impending respiratory failure, selective patients may benefit from rescue NPPV.
\end{abstract}

\section{Introduction}

The incidence of extubation failure and reintubation in critically ill patients is approximately $10 \%$ to $30 \%$, which leads to high mortality rate after weaning from mechanical ventilation (MV) $[1,2]$. Studies showed that not only preexisting heart failure but also cardiac etiology for respiratory failures such as systolic heart failure, acute coronary syndrome, and severe valvular heart disease were high risks for extubation failure [3, 4]. Moreover, cardiac function and left ventricular ejection fracture (LVEF) were strongly associated with extubation failure [5]. Therefore, in the postextubation period in which cardiac performance is still in recovery, reducing the work of the cardiopulmonary system 
to prevent extubation failure in patients with heart failure is extremely important.

Positive end-expiratory pressure (PEEP) during invasive mechanical ventilation is one of the nonpharmacological approaches beneficial for heart failure caused by an increase in the intrathoracic pressure [6]. First, PEEP can augment cardiac output through a decrease in the right ventricular preload, left ventricular preload, and afterload [6]. Second, PEEP can counterbalance the hydrostatic force and decrease the extravascular lung water by exerting pressure at the level of the alveoli and interstitium [6]. Third, PEEP helps maintain alveolar pressure to prevent alveolar collapse [7]. However, end positive airway pressure (EPAP) is used for noninvasive ventilation and generates hemodynamic effects in cases of heart failure, similar to continuous positive airway pressure (CPAP) delivered by a face mask [7], which increases cardiac performance and stroke volume in patients with heart failure and a high left ventricular filling pressure [8-10]. Two noninvasive respiratory devices can generate positive pressure during the expiratory phase and have been used to prevent extubation failure, namely, high-flow nasal cannula (HFNC) and noninvasive positive pressure ventilation (NPPV).

HFNC is characterized by the stable concentration of oxygen and variable PEEP, which might have potential benefits for patients with heart failure [11]. A study found that HFNC could reduce the inspiratory inferior vena cava (IVC) collapse and right heart preload [12]. Further, HFNC could relieve dyspnea and hypoxemia in cardiogenic pulmonary edema $[13,14]$.

In the past decade, NPPV was extensively used for preventing extubation failure in high-risk patients, and this was proved with a moderate level of evidence $[15,16]$. Two large randomized controlled trials demonstrated that NPPV could be applied for the prevention of postextubation respiratory failure in patients at risk of extubation failure, including those with heart failure $[17,18]$. Recently, HFNC was increasingly used after scheduled extubation as an alternative therapy of NPPV because of its ease of application, patient tolerance, physiological benefits, and lower adverse events $[19,20]$. Randomized studies showed that HFNC was not inferior to NPPV for preventing extubation failure in general high-risk extubation failure and postcardiothoracic surgery patients $[21,22]$.

However, in patients with heart failure, to the best of our knowledge, no studies have compared the potential benefits obtained with NPPV and those with HFNC after extubation. Therefore, this retrospective study aimed to compare the effectiveness of HFNC and NPPV for preventing extubation failure in critically ill patients with heart failure.

\section{Materials and Methods}

2.1. Study Design. A retrospective observational cohort study was conducted between January 2015 and January 2018 at a medical center with four adult intensive care units (ICUs) in New Taipei City, Taiwan. This study was approved by the Ethics Committee of Taipei Medical University
(N201808011), and informed consent was waived because of its observational nature.

2.2. Patient Selection. We reviewed a total of 5,497 ICU patients who had received MV for tracheal intubation and included 134 patients according to the following criteria: patients (1) who were on mechanical ventilator with endotracheal tube for more than $24 \mathrm{~h}$, (2) who passed the weaning readiness assessment, (3) who had first episode of extubation, (4) who had heart failure with LVEF $<50 \%$ determined by echocardiography under M-mode or Simpson's rule in the apical four-chamber view before extubation, and (5) on whom prophylactic HFNC or NPPV was applied as first-line therapy after extubation. The exclusion criteria were tracheostomy, ventilator dependence status before admission, self-extubation, palliative extubation, and donot-reintubate status. After implementing these criteria, a total of 104 patients were recruited for the analysis (Figure 1).

All patients have received the following standard ventilator bundle: (1) head and bed elevation at least $30^{\circ}$ to $45^{\circ}$, (2) oral care with $0.12 \%-0.2 \%$ chlorhexidine at least twice a day, and (3) daily sedation interruption and screening for weaning from ventilator. The weaning readiness assessment evaluated by respiratory therapists and physicians included (1) recovery from the reason of intubation, (2) hemodynamic stability or administration of low-dose inotropic agent, (3) no sedation or low-dose sedation with RASS $\geq-1$, (4) arterial blood gas sample, (5) weaning parameters, (6) spontaneous breathing trial (SBT), and (7) evaluation of airway patency and secretions.

Device selection after extubation followed a patientoriented care concept. On the premise that noninvasive respiratory support devices (HFNC or NPPV) were available, we consistently applied these devices as postextubation preventive strategies for patients with heart failure and with LVEF $<50 \%$. Before extubation, respiratory therapists and intensivists would assess and decide which devices (HFNC or NPPV) should be applied to patients. The decision to provide HFNC depended on the following assessments: (1) risk of aspiration, (2) facial trauma, (3) claustrophobia, (4) excessive airway secretions or saliva that required suction twice per hour, and (5) patients could not tolerate mask ventilation and refuse it. If providing HFNC did not meet the above criteria, NPPV with stable positive airway pressure was first applied to patients as respiratory support after extubation.

2.3. Outcomes. Patients who received prophylactic HFNC as first-line therapy were compared with those who received prophylactic NPPV alone. The primary outcome was treatment failure within 72 hours after extubation. HFNC failure was defined as escalation to use of other respiratory devices including NPPV or invasive MV within 72 hours. NPPV failure was defined as the need for invasive MV within 72 hours. Secondary outcomes included reintubation within 72 hours, reintubation, ICU and hospital lengths of stay (LOS), and ICU and hospital mortality. 


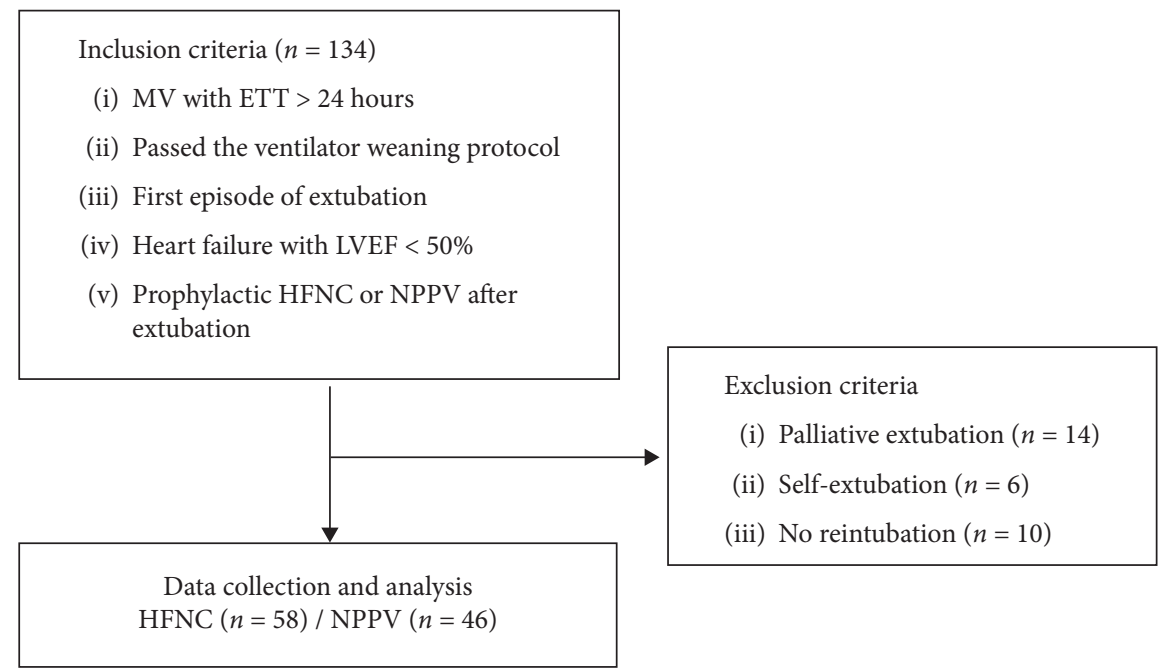

FIGURE 1: Retrospective flow chart of patients included in analyses between January 2015 and January 2018. ETT, endotracheal tube; HFNC, high-flow nasal cannula; MV, mechanical ventilation; NPPV, noninvasive positive pressure ventilation.

2.4. Data Collection. The following characteristics were collected: age, sex, body mass index (BMI), smoking status, Acute Physiology and Chronic Health Evaluation II (APACHE II) score upon ICU admission, echocardiographic data, diagnosis at admission, comorbidities, weaning status (includes rapid shallow breathing index, maximum inspiratory pressure, maximum expiratory pressure, cuff leak test, MV days before extubation, Glasgow coma scale on extubation day), and the setting of HFNC and NPPV. Treatment failure was determined on the basis of arterial blood gas $(\mathrm{ABG})$ data and/or symptoms reported in the medical records and classified as follows: (1) hypoxemic respiratory failure, defined as peripheral capillary oxygen saturation $\left(\mathrm{SpO}_{2}\right)<90 \%$ or arterial partial pressure of oxygen $\left(\mathrm{PaO}_{2}\right)<60 \mathrm{mmHg}$ with fraction of inspired oxygen $\left(\mathrm{FiO}_{2}\right)>0.5$; (2) hypercapnic respiratory failure, defined as $\mathrm{pH}<7.30$ with arterial partial pressure of carbon dioxide $\left(\mathrm{PaCO}_{2}\right)>50 \mathrm{mmHg}$; (3) excessive effort for breathing, defined as respiratory rate $>35$ breaths per minute and/or the use of accessory muscles and paradoxical respiration; (4) hemodynamic compromise, defined as cardiac arrest, bradycardia with loss of alertness, and/or severe hemodynamic instability; and (5) airway protection, defined as the persistent inability to remove respiratory secretions, upper airway obstruction, massive aspiration, and/or upper gastrointestinal bleeding. Pre-extubation ABG data for the patients with treatment failure were collected in order to confirm that they received prophylactic noninvasive respiratory devices under stable conditions. We determined the types of postextubation respiratory failure from the ABG data, with the exception of three types that could be identified by the findings of physical assessments mentioned in the medical records.

2.5. Statistical Analysis. Descriptive statistics were expressed as mean \pm standard deviation (SD), median (Q1-Q3), or number (percentages). The chi-squared or Fisher's exact test was used to compare categorical variables. The
Mann-Whitney $U$ test was used to compare continuous variables between two independent samples. Additional univariate analyses for primary outcomes and multivariate logistic regression were performed to account for clinical confounding factors and statistical differences. We selected variables in the univariate analysis with $p$ value $<0.20$ and included them in the multivariate logistic regression. Considering the baseline differences between the groups, a propensity score was computed by using logistic regression with the baseline variables (baseline characteristics, echocardiography data, comorbidities, weaning parameters, and diagnosis). A matching method was performed using the propensity scores. A two-tailed $p$ value $<0.05$ was considered significant. Statistical analyses were performed using the statistical software IBM SPSS version 22 (IBM Corp., Armonk, NY, USA) and R statistical package (R Foundation for Statistical Computing, Vienna, Austria).

\section{Results}

A total of 104 patients were enrolled and analyzed in this study, including 58 in the HFNC group and 46 in the NPPV group. Clinical characteristics, echocardiographic data, diagnosis, comorbidities, disease severity, and weaning status in the two groups were similar. Among all patients, the median LVEF was $34.1 \%(27.3 \%-41.2 \%)$ and $72.1 \%$ of the patients were classified as having systolic heart failure (LVEF $<40 \%)$. The most common diagnosis upon admission was pneumonia, acute decompensated heart failure with pulmonary edema, and extrapulmonary sepsis, which were also similar between the two groups (Table 1).

The primary outcome, i.e., treatment failure within 72 hours, occurred in 15 (25.9\%) patients with HFNC and six (13\%) patients with NPPV, and no significant difference was noted between the two groups $(p=0.106)$. However, the two groups differed with regard to the type of treatment failure, with the rate of hypoxemic respiratory failure within 72 hours being significantly lower in the NPPV group than in 
TABLE 1: Baseline characteristics of patients in the HFNC and NPPV groups.

\begin{tabular}{|c|c|c|c|}
\hline Characteristics & HFNC $(n=58)$ & NPPV $(n=46)$ & $p$ value \\
\hline Age, years & $74.5(63.8-82.3)$ & $72.5(61.8-79)$ & 0.366 \\
\hline Male, $n$ & $37(63.8)$ & $28(60.9)$ & 0.76 \\
\hline Smoke, $n$ & $24(41.4)$ & $18(39.1)$ & 0.816 \\
\hline $\mathrm{BMI}, \mathrm{kg} / \mathrm{m}^{2}$ & $23.03(19.7-25.1)$ & $23.39(20.9-27.3)$ & 0.214 \\
\hline APACHE II at ICU admission, point & $21(16.8-27)$ & $24(18-30)$ & 0.074 \\
\hline \multicolumn{4}{|l|}{ Echocardiography } \\
\hline LVEF, \% & $33.55(26.6-41.2)$ & $35.4(27.9-41.1)$ & 0.456 \\
\hline Systolic heart failure $(\mathrm{LVEF}<40 \%), n$ & $42(72.4)$ & $33(71.1)$ & 0.939 \\
\hline Moderate to severe VHD, $n$ & $26(44.8)$ & $25(54.3)$ & 0.335 \\
\hline \multicolumn{4}{|l|}{ Diagnosis of admission } \\
\hline Cardiac arrest, $n$ & $4(6.9)$ & $3(6.5)$ & $>0.99$ \\
\hline Pneumonia, $n$ & $25(43.1)$ & $15(32.6)$ & 0.275 \\
\hline Exacerbated COPD, $n$ & $1(1.7)$ & $2(4.3)$ & 0.582 \\
\hline Acute decompensated heart failure, $n$ & $11(19)$ & $14(30.4)$ & 0.174 \\
\hline Acute coronary syndrome, $n$ & $4(6.9)$ & $4(8.7)$ & 0.730 \\
\hline Extrapulmonary sepsis, $n$ & $8(13.8)$ & $4(8.7)$ & 0.419 \\
\hline Cardiac-thoracic surgery, $n$ & $1(1.7)$ & $2(4.3)$ & 0.582 \\
\hline Others: emergency surgery, $n$ & $1(1.7)$ & $2(4.3)$ & 0.582 \\
\hline Others, $n$ & $3(5.2)$ & 0 & 0.253 \\
\hline \multicolumn{4}{|l|}{ Comorbidities } \\
\hline Hypertension, $n$ & $39(67.2)$ & $37(80.4)$ & 0.132 \\
\hline Coronary artery disease, $n$ & $27(46.6)$ & $19(41.3)$ & 0.593 \\
\hline Cerebrovascular disease, $n$ & $13(22.4)$ & $9(19.6)$ & 0.724 \\
\hline Obstructive lung disease, $n$ & $9(15.5)$ & $11(23.9)$ & 0.281 \\
\hline Diabetes mellitus, $n$ & $36(62.1)$ & $22(47.8)$ & 0.146 \\
\hline Chronic kidney disease, $n$ & $24(41.1)$ & $12(26.1)$ & 0.104 \\
\hline Cancer, $n$ & $6(10.3)$ & $3(6.5)$ & 0.728 \\
\hline \multicolumn{4}{|l|}{ Weaning status } \\
\hline RSBI, cycles/min/L & $59(39.1-77.8)$ & $74.65(51.2-99.3)$ & 0.055 \\
\hline Pimax, $\mathrm{cmH} 2 \mathrm{O}$ & $40(36-55.5)$ & $48(40-64)$ & 0.1 \\
\hline Pemax, $\mathrm{cmH} 2 \mathrm{O}$ & $50(36-80)$ & $68(40-118)$ & 0.135 \\
\hline Minute ventilation, $\mathrm{L} / \mathrm{min}$ & $9(7.2-10.7)$ & $8.33(6.1-10.6)$ & 0.371 \\
\hline Cuff leak test, $\mathrm{ml}$ & $284(184.8-352.5)$ & $246(131.5-346.5)$ & 0.286 \\
\hline Duration of MV before extubation, days & $9.5(5.8-14)$ & $8.5(5-12)$ & 0.539 \\
\hline GCS in the day of extubation, score & $14(13-15)$ & $15(13-15)$ & 0.057 \\
\hline \multicolumn{4}{|l|}{ Initial setting of HFNC/NPPV } \\
\hline HFNC flow, L/min & $49.4 \pm 6.15$ & & \\
\hline NPPV inspiratory pressure, $\mathrm{cmH} 2 \mathrm{O}$ & & $13.43 \pm 2.55$ & \\
\hline NPPV PEEP level, cmH2O & & $7.3 \pm 0.96$ & \\
\hline Initial $\mathrm{FiO}_{2}, \%$ & $41.91 \pm 9.71$ & $35.07 \pm 4.94$ & 0.449 \\
\hline The length of HFNC/NPPV use, hours & $25.5(20-68.5)$ & $20.5(2.75-71.75)$ & 0.149 \\
\hline
\end{tabular}

Values are mean \pm SD, median (Q1-Q3), or number (percentage \%). APACHE II, Acute Physiology and Chronic Health Evaluation II; BMI, body mass index; COPD, chronic obstructive pulmonary disease; $\mathrm{FiO}_{2}$, fraction of inspired oxygen; GCS, Glasgow Coma Scale; LVEF, left ventricular ejection fraction; HFNC, high-flow nasal cannula; MV, mechanical ventilation; NPPV, non-invasive positive pressure ventilation; PEEP, positive end expiatory pressure; Pemax, maximum expiratory pressure; Pimax, maximum inspiratory pressure; RSBI, rapid shallow breathing index; VHD, valvular heart disease * $p$ value $<0.05$.

the HFNC group ( $p=0.046$ ) (Table 2). According to the ABG data, the cause of the five types of treatment failure could be deterioration of the primary disease or underlying disease or inadequate respiratory support (Supplement 1). Secondary outcomes, including reintubation within 72 hours, reintubation, ICU and hospital LOS, and ICU and hospital mortality, were not significantly different between the two groups (Table 2). Even after matching, there was no significant between-group difference in terms of treatment failure within 72 hours $(p=0.664)$ (Supplement 2).

In the HFNC group, eight patients required immediate reintubation once treatment failure developed, and the other seven patients used NPPV as rescue therapy. Rescue NPPV successfully avoided reintubation in five patients, but it failed in two patients. In the NPPV group, six patients needed reintubation because of treatment failure (Figure 2). The reintubation rate of the NPPV group increased sharply 1 week after extubation, even if the reintubation rate was higher in HFNC previously. However, the reintubation rate was not significantly different between the two groups in the Kaplan-Meier plot ( $\mathrm{p}$ of the log-rank test $=0.925$ ) (Figure 3 ).

The results of the univariate analysis and multivariate logistic regression for variables associated with treatment failure within 72 hours are shown in Table 3. Variables in the univariate analysis with $p$ value $<0.20$ were HFNC vs NPPV (HFNC as first-line therapy compared with NPPV alone), 
TABLE 2: Outcomes of patients between the HFNC and NPPV groups.

\begin{tabular}{|c|c|c|c|}
\hline Outcomes & HFNC $(n=58)$ & $\operatorname{NPPV}(n=46)$ & $p$ value \\
\hline \multicolumn{4}{|l|}{ Primary outcome } \\
\hline $\begin{array}{l}\text { Treatment failure within } 72 \text { hours, } n \\
\text { Type of treatment failure }\end{array}$ & $15(25.9)$ & $6(13)$ & 0.106 \\
\hline Hypoxemia respiratory failure, $n$ & $11(73.3)$ & $1(16.7)$ & $0.046^{*}$ \\
\hline Hypercapnia respiratory failure, $n$ & $3(20)$ & $2(33.3)$ & 0.598 \\
\hline Excessive effort for breathing, $n$ & 0 & $1(16.7)$ & 0.286 \\
\hline Cardiac arrest, $n$ & $1(6.7)$ & 0 & $>0.99$ \\
\hline Airway protection, $n$ & 0 & $2(33.3)$ & 0.71 \\
\hline Time to treatment failure, hours & $17(4-54)$ & $36(4.5-49.8)$ & 0.726 \\
\hline \multicolumn{4}{|l|}{ Secondary outcome } \\
\hline Reintubation within 72 hours, $n$ & $10(17.2)$ & $6(13)$ & 0.556 \\
\hline Hospital reintubation, $n$ & $17(29.3)$ & $14(30.4)$ & 0.901 \\
\hline Time to reintubation, hours & $69(14-274)$ & $145(40.5-221.3)$ & 0.691 \\
\hline ICU length of stay, days & $14(8-21.8)$ & $11(8-16.3)$ & 0.138 \\
\hline Hospital length of stay, days & $23.5(16-46.3)$ & $27(18.5-38)$ & 0.883 \\
\hline ICU mortality, $n$ & $1(1.7)$ & $1(2.2)$ & $>0.99$ \\
\hline Hospital mortality, $n$ & $3(5.2)$ & $8(17.4)$ & 0.057 \\
\hline
\end{tabular}

Values are mean $\pm S D$, median $(\mathrm{Q} 1-\mathrm{Q} 3)$, or number (percentage \%); ICU, intensive care unit; HFNC, high-flow nasal cannula; NPPV, noninvasive positive pressure ventilation. ${ }^{*} p$ value $<0.05$.

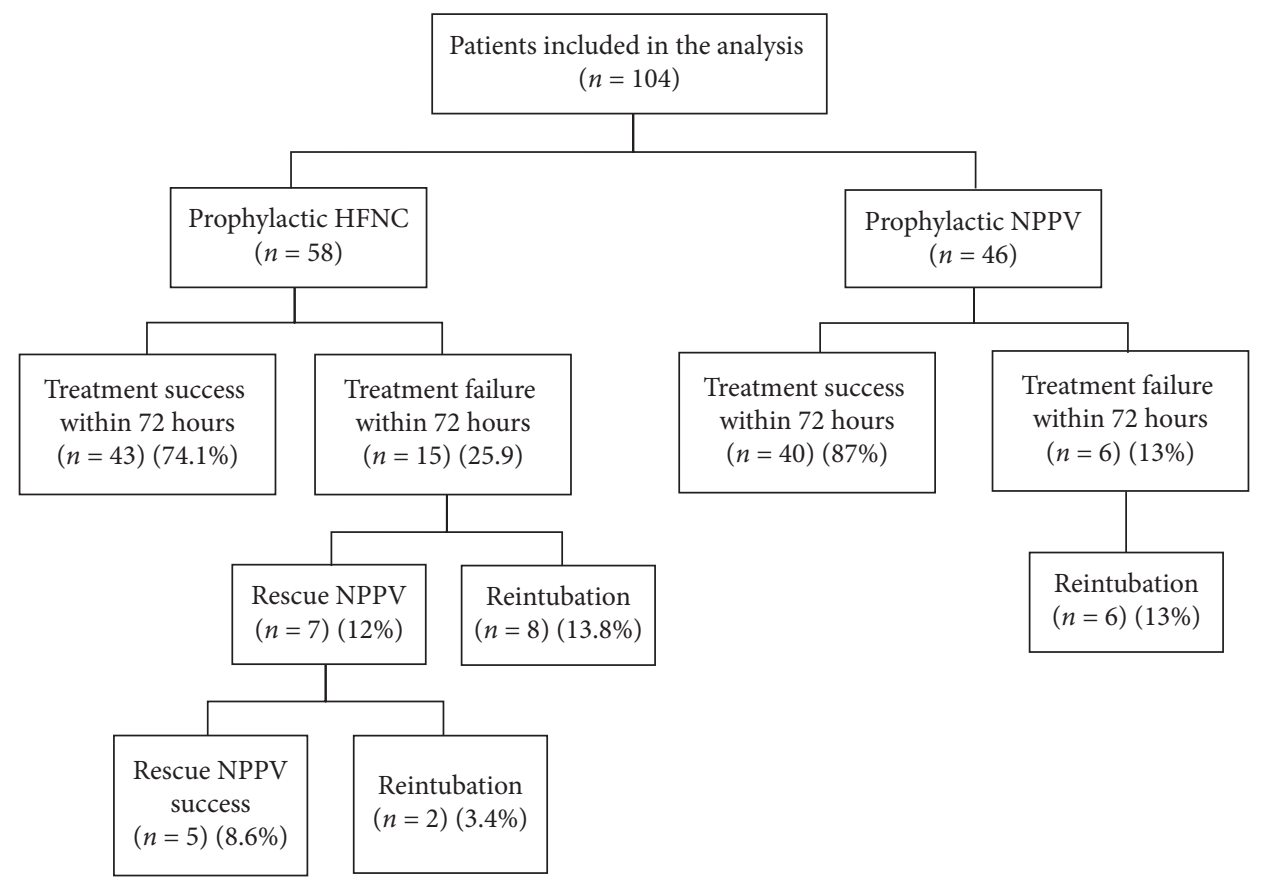

FIGURE 2: Outcomes following extubation within 72 hours. In the HFNC group, eight patients required immediate reintubation once treatment failure developed, and the other seven patients used NPPV as rescue therapy. Rescue NPPV successfully avoided reintubation in five patients, but it failed in two patients. In the NPPV group, six patients needed reintubation because of treatment failure. HFNC, highflow nasal cannula; NPPV, noninvasive positive pressure ventilation.

admission diagnosis of pneumonia, and chronic kidney disease as underlying comorbidity. The above three variables were included in the multivariate logistic regression, and no independent variables were associated with treatment failure within 72 hours (Table 3 ).

\section{Discussion}

In this study, no significant difference exists between prophylactic HFNC as first-line therapy and prophylactic NPPV alone in preventing extubation failure in critically ill patients with heart failure.

We found that HFNC has been applied more in patients with heart failure than NPPV in the past 3 years because of its better comfort, patient tolerance, and easier assembly, which was different from the past practice of using prophylactic NPPV alone for such high-risk extubation failure population. Moreover, in this study, no significant difference was found between HFNC and NPPV in preventing treatment failure, which might be due to the following features of 


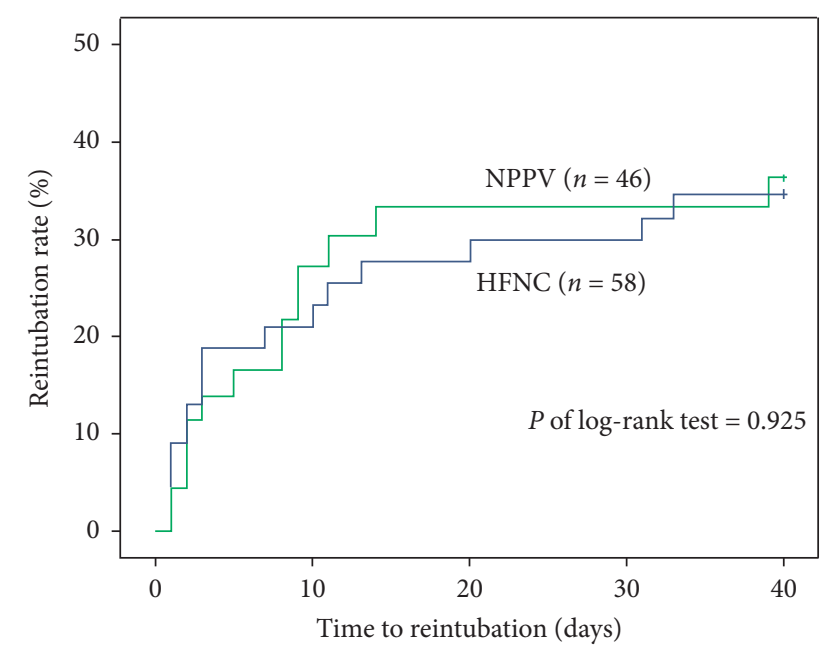

Figure 3: Kaplan-Meier plot of time to reintubation. HFNC, highflow nasal cannula; NPPV, noninvasive positive pressure ventilation.

HFNC. First, the high-flow rate from the cannula against the expiratory airflow from patients generates the PEEP effect. Okuda et al. [23] confirmed the PEEP effect of HFNC by evaluating the correlation of end-expiratory esophageal pressure and flow rate. In this study, the initial flow of HFNC was $49.4 \pm 6.15 \mathrm{~L} / \mathrm{min}$, and a previous study has shown that such high flow could generate a certain degree of PEEP [24]. Mauri et al. [25] demonstrated that HFNC setting greater than $40 \mathrm{~L} / \mathrm{min}$ could improve dynamic lung compliance and reduce inspiratory effort and metabolic work of breathing, which represented external ventilation support. Roca et al. [12] found that the flow of HFNC increased and the IVC collapse decreased, which confirmed that HFNC reduced the cardiac preload. Furthermore, Makdee et al. [13] found that HFNC reduced the respiratory rate in patients with acute cardiogenic pulmonary edema.

Second, the heated and humidified flow facilitated secretion clearance [11]. In this study, $38 \%$ of the patients were admitted to the hospital due to pneumonia; hence, it was necessary to maintain airway clearance after extubation. Excessive secretion was one of the major causes of extubation failure [26], and HFNC might reduce sputum retention to improve outcomes for pneumonia patients [27]. A recent meta-analysis suggested that pneumonia patients might benefit from the physiological effects of HFNC, which could reduce ICU mortality [28]. In the present study, even though the multivariate logistic regression analysis showed that pneumonia tended to associate with treatment failure, HFNC was not inferior to NPPV as a prophylactic strategy as there are more pneumonia patients in the HFNC group.

Third, HFNC is characterized by higher patient comfort and tolerance; thus, its continued application was easier $[22,29]$. In this study, HFNC was continuously administered to patients, but NPPV was administered intermittently due to patient compliance. Thus, NPPV was unable to provide sustained noninvasive respiratory support after extubation. Based on the above perspectives, we believe that HFNC might reduce the metabolism and load of respiratory muscles and provide adequate non-invasive respiratory support after extubation.

NPPV has been proven beneficial for heart failure by preventing alveolar collapse and counterbalancing alveolar fluids [30, 31]. Therefore, adequate threshold of PEEP and positive inspiratory pressure (PIP) supplied by NPPV might benefit some patients in this study. Three findings can support the above hypothesis. First, even if there was no significant difference in treatment failure between the HFNC and NPPV, treatment failure occurred half as often in the NPPV group. Furthermore, the incidence of hypoxemia respiratory failure was significantly lower in the NPPV group than in the HFNC group, which is consistent with the observation of our previous study that NPPV reduces postextubation respiratory failure caused by heart failure [26]. Previous studies also reported that better oxygenation with NPPV was probably due to higher PIP and PEEP compared with HFNC $[21,32]$. Second, in the HFNC group, seven patients who received rescue NPPV had a worse cardiac function (median LVEF $=25.9 \%$ ) than overall patients, but five of the seven patients were successfully weaned from the NPPV (Figure 2). Third, the reintubation rate increased sharply after 1 week in the NPPV group, which might be related to the withdrawal of NPPV after 72 hours (Figure 3). Accordingly, HFNC was similar to NPPV in preventing extubation failure, but NPPV might benefit selective patients with heart failure. Further research is needed to identify more accurate cardiac function indicators as standard for NPPV application.

A recent prospective study [33] reported that prophylactic NPPV reduced the 72-hour reintubation rate from $24 \%$ to $13 \%$ in patients aged $>65$ years and with underlying cardiac and respiratory disease, which was similar to our study population and reintubation rate. In our study, the reintubation rate within 72 hours in both groups (HFNC $17.2 \%$ vs NPPV 13\%) were similar to that in Stéphan et al.'s study [21] (HFNC 14\% vs NPPV 13.7\%) and lower than those in Hernández et al.'s study [22] (HFNC 22.8\% vs NPPV 19.1\%). Even though our study included patients with more severe (median APACHE II =22), worse cardiac function (median $\mathrm{LEVF}=34.4 \%$ ) and longer MV duration prior to extubation (median MV days =9), the reintubation rate within 72 hours was lower than those in Hernández et al.'s study, which was due to two reasons. First, in the actual clinical setting, rescue NPPV was allowed when HFNC failed or postextubation respiratory failure occurred, and some patients benefited from rescue NPPV to reduce the reintubation rate. Second, the positive airway pressure and cardiopulmonary support provided by HFNC and NPPV might be beneficial to patients with cardiac dysfunction. Two randomized controlled trials demonstrated that NPPV reduces the rate of reintubation, which lead to a reduced ICU mortality in high-risk extubation patients $[17,18]$. In this study, ICU mortality was relatively low in both devices, indicating the effectiveness of both in the period of postextubation. The hospital mortality rate was different between groups after the propensity score matching, but given the small sample size, further RCT is warranted. 
TABLE 3: Univariate and multivariate analyses of variables associated with treatment failure and hospital mortality.

\begin{tabular}{|c|c|c|c|c|}
\hline \multirow{3}{*}{ Variables } & \multicolumn{4}{|c|}{ Treatment failure within 72 hours } \\
\hline & \multicolumn{2}{|c|}{ Univariate analysis } & \multicolumn{2}{|c|}{ Multivariate analysis } \\
\hline & OR $(95 \% \mathrm{CI})$ & $p$ value & Adjusted OR (95\% CI) & $p$ value \\
\hline \multicolumn{5}{|l|}{ Characteristics } \\
\hline HFNC vs NPPV & $2.326(6.58-0.822)$ & $0.112^{\S}$ & $2.004(0.687-5.841)$ & 0.204 \\
\hline Reintubation & - & & - & - \\
\hline Age (years) & $0.982(0.952-1.014)$ & 0.268 & - & - \\
\hline Male & $0.969(0.361-2.598)$ & 0.95 & - & - \\
\hline Smoke & $0.887(0.332-2.371)$ & 0.811 & - & - \\
\hline BMI, $\mathrm{kg} / \mathrm{m}^{2}$ & $0.945(0.863-1.034)$ & 0.215 & - & - \\
\hline APACHE II at ICU admission, score & $0.969(0.906-1.037)$ & 0.363 & - & - \\
\hline \multicolumn{5}{|l|}{ Echocardiography } \\
\hline LVEF, \% & $1.011(0.956-1.069)$ & 0.704 & - & - \\
\hline Systolic heart failure & $0.958(0.331-2.771)$ & 0.937 & - & - \\
\hline Moderate to severe VHD & $1.182(0.453-3.084)$ & 0.732 & - & - \\
\hline \multicolumn{5}{|l|}{ Diagnosis of admission } \\
\hline Cardiac arrest & - & & - & - \\
\hline Pneumonia & $2.619(0.986-6.956)^{\S}$ & $0.053^{\S}$ & $2.645(0.968-7.227)$ & 0.053 \\
\hline Exacerbated COPD & $2.025(0.175-23.463)$ & 0.572 & - & - \\
\hline Acute decompensated heart failure & $1.806(0.634-5.143)$ & 0.269 & - & - \\
\hline Acute coronary syndrome & - & & - & - \\
\hline Extrapulmonary sepsis & $0.327(0.04-2.689)$ & 0.299 & - & - \\
\hline \multicolumn{5}{|l|}{ Comorbidities } \\
\hline Hypertension & $0.677(0.241-1.904)$ & 0.46 & - & - \\
\hline Coronary artery disease & $0.564(0.207-1.540)$ & 0.264 & - & - \\
\hline Cerebrovascular disease & $0.85(0.254-2.843)$ & 0.792 & - & - \\
\hline Obstructive lung disease & $1.417(0.449-4.471)$ & 0.553 & - & - \\
\hline Diabetes mellitus & $0.843(0.323-2.201)$ & 0.727 & - & - \\
\hline Chronic kidney disease & $1.993(0.753-5.278)^{\S}$ & $0.165^{\S}$ & $1.983(0.718-5.475)$ & 0.186 \\
\hline \multicolumn{5}{|l|}{ Weaning status } \\
\hline RSBI, cycles/min/L & $1.005(0.99-1.021)$ & 0.512 & - & - \\
\hline Pimax, $\mathrm{cmH} 2 \mathrm{O}$ & $1.008(0.979-1.038)$ & 0.6 & - & - \\
\hline Pemax, $\mathrm{cmH} 2 \mathrm{O}$ & $1.006(0.992-1.021)$ & 0.385 & - & - \\
\hline Minute ventilation, $\mathrm{L} / \mathrm{min}$ & $0.976(0.836-1.139)$ & 0.757 & - & - \\
\hline Cuff leak test, ml & $1.0(0.996-1.003)$ & 0.958 & - & - \\
\hline MV days before extubation, days & $1.036(0.97-1.106)$ & 0.293 & - & - \\
\hline GCS in the day of extubation, score & $0.913(0.731-1.139)$ & 0.419 & - & - \\
\hline
\end{tabular}

APACHE II, Acute Physiology and Chronic Health Evaluation II; BMI, body mass index; CI, confidence interval; COPD, chronic obstructive pulmonary disease; GCS, Glasgow Coma Scale; LVEF, left ventricular ejection fraction; HFNC, high-flow nasal cannula; MV, mechanical ventilation; NPPV, noninvasive positive pressure ventilation; OR, odds ratio; Pemax, maximum expiratory pressure; Pimax, maximum inspiratory pressure; RSBI, rapid shallow breathing index; VHD, valvular heart disease. ${ }^{\$} p$ value $<0.2$ in the univariate analysis. ${ }^{*} p$ value $<0.05$ in the multivariate analysis.

The European Respiratory Society and American Thoracic Society clinical practice guidelines recommended that NPPV be used to prevent extubation failure in high-risk populations, despite the low certainty of evidence [34]. We could not exclude that NPPV is still an effective strategy for preventing extubation failure in patients with heart failure. However, from the perspective of preventing extubation failure, we considered that HFNC could be another alternative treatment for patients with underlying cardiac disease because of its ease of application and physiological benefits over conventional oxygen therapy [25], and our data demonstrated the effectiveness and safety of HFNC after extubation.

To our knowledge, no studies have investigated the effectiveness of HFNC and NPPV in critically ill patients with heart failure after extubation. The results of our data analysis were also consistent with our clinical experience.
Nevertheless, this study has some limitations because of its retrospective nature. First, selection bias might not be completely avoided. Patients who were excluded from the analysis due to "palliative extubation" and "do not reintubation" status had poor outcomes, though the baseline characteristics were similar between the two cohorts. Second, the decision of using preventive respiratory devices was patient compliance-oriented, but there were no definite criteria for the assessment. Third, the clinical decision was in favor of applying HFNC to patients with excessive airway secretions that required frequent suctioning, and this may have caused bias. Fourth, the lack of physiological parameters directly confirmed the benefits of the two devices. Fifth, echocardiography was performed within a period before extubation, so it was difficult to accurately describe patients' cardiac function immediately during extubation. Besides, this study was conducted in one medical center with a small 
size sample. We look forward to a future prospective study or randomized controlled trial to explore the physiologic benefits and clinical outcomes of these two devices for patients with heart failure.

\section{Conclusions}

In critically ill patients with heart failure, there was no significant difference in the effectiveness of prophylactic HFNC in preventing extubation failure compared with NPPV. We suggest that prophylactic HFNC can be applied as first-line therapy after extubation. However, in case of an impending respiratory failure, selective patients may benefit from rescue NPPV.

\section{Conflicts of Interest}

The authors declare that there are no conflicts of interest.

\section{Acknowledgments}

The authors would like to acknowledge all their colleagues in the Department of Chest Medicine and Respiratory Therapy at Taipei Medical University-Shuang Ho Hospital, especially Hsin-I Tsai, for sharing suggestions and experiences during the research process. In addition, the authors would like to thank Dr. Yan-guang Lin from Taipei Medical University for his help in the statistical analysis.

\section{Supplementary Materials}

Supplement 1: arterial blood gas (ABG) data before extubation and at the time of treatment failure in patients with treatment failure. Supplement 2: baseline characteristics and outcomes between propensity score-matched patients in HFNC and NPPV groups. (Supplementary Materials)

\section{References}

[1] A. Esteban, F. F. Vivar, A. Muriel et al., "Evolution of mortality over time in patients receiving mechanical ventilation," American Journal of Respiratory and Critical Care Medicine, vol. 188, no. 2, pp. 220-230, 2013.

[2] A. W. Thille, J.-C. M. Richard, and L. Brochard, "The decision to extubate in the intensive care unit," American Journal of Respiratory and Critical Care Medicine, vol. 187, no. 12, pp. 1294-1302, 2013.

[3] S. K. Epstein, R. L. Ciubotaru, and J. B. Wong, "Effect of failed extubation on the outcome of mechanical ventilation," Chest, vol. 112, no. 1, pp. 186-192, 1997.

[4] A. W. Thille, A. Harrois, F. Schortgen, C. Brun-Buisson, and L. Brochard, "Outcomes of extubation failure in medical intensive care unit patients*," Critical Care Medicine, vol. 39, no. 12, pp. 2612-2618, 2011.

[5] A. W. Thille, F. Boissier, H. Ben Ghezala, K. Razazi, A. Mekontso-Dessap, and C. Brun-Buisson, "Risk factors for and prediction by caregivers of extubation failure in ICU patients," Critical Care Medicine, vol. 43, no. 3, pp. 613-620, 2015.

[6] T. Luecke and P. Pelosi, "Clinical review: positive end-expiratory pressure and cardiac output," Critical Care, vol. 9, no. 6, pp. 607-621, 2005.
[7] T. Kato, S. Suda, and T. Kasai, "Positive airway pressure therapy for heart failure," World Journal of Cardiology, vol. 6, no. 11, pp. 1175-1191, 2014.

[8] A. De Hoyos, P. P. Liu, D. C. Benard, and T. D. Bradley, "Haemodynamic effects of continuous positive airway pressure in humans with normal and impaired left ventricular function," Clinical Science, vol. 88, no. 2, pp. 173-178, 1995.

[9] S. Steiner, C. M. Schannwell, and B. E. Strauer, "Left ventricular response to continuous positive airway pressure: role of left ventricular geometry," Respiration, vol. 76, no. 4, pp. 393-397, 2008.

[10] M. Yoshida, T. Kadokami, H. Momii et al., "Enhancement of cardiac performance by bilevel positive airway pressure ventilation in heart failure," Journal of Cardiac Failure, vol. 18, no. 12, pp. 912-918, 2012.

[11] M. Nishimura, "High-flow nasal cannula oxygen therapy in adults: physiological benefits, indication, clinical benefits, and adverse effects," Respiratory Care, vol. 61, no. 4, pp. 529-541, 2016.

[12] O. Roca, P. Pérez-Terán, J. R. Masclans et al., "Patients with New York Heart Association class III heart failure may benefit with high flow nasal cannula supportive therapy," Journal of Critical Care, vol. 28, no. 5, pp. 741-746, 2013.

[13] O. Makdee, A. Monsomboon, U. Surabenjawong et al., "Highflow nasal cannula versus conventional oxygen therapy in emergency department patients with cardiogenic pulmonary edema: a randomized controlled trial," Annals of Emergency Medicine, vol. 70, no. 4, pp. 465-472, 2017.

[14] N. Rittayamai, J. Tscheikuna, N. Praphruetkit, and S. Kijpinyochai, "Use of high-flow nasal cannula for acute dyspnea and hypoxemia in the emergency department," Respiratory Care, vol. 60, no. 10, pp. 1377-1382, 2015.

[15] A. Demoule, A. Carlucci, S. Chevret et al., "Changing use of noninvasive ventilation in critically ill patients: trends over 15 years in francophone countries," Intensive Care Medicine, vol. 42, no. 1, pp. 82-92, 2016.

[16] D. R. Ouellette, S. Patel, T. D. Girard et al., "Liberation from mechanical ventilation in critically ill adults: an official American college of chest physicians/American thoracic society clinical practice guideline," Chest, vol. 151, no. 1, pp. 166-180, 2017.

[17] S. Nava, C. Gregoretti, F. Fanfulla et al., "Noninvasive ventilation to prevent respiratory failure after extubation in highrisk patients*," Critical Care Medicine, vol. 33, no. 11, pp. 2465-2470, 2005.

[18] M. Ferrer, M. Valencia, J. M. Nicolas, O. Bernadich, J. R. Badia, and A. Torres, "Early noninvasive ventilation averts extubation failure in patients at risk," American Journal of Respiratory and Critical Care Medicine, vol. 173, no. 2, pp. 164-170, 2006.

[19] O. Roca, G. Hernández, S. Díaz-Lobato et al., "Current evidence for the effectiveness of heated and humidified high flow nasal cannula supportive therapy in adult patients with respiratory failure," Critical Care, vol. 20, no. 1, p. 109, 2016.

[20] C. C. Lee, D. Mankodi, S. Shaharyar et al., "High flow nasal cannula versus conventional oxygen therapy and non-invasive ventilation in adults with acute hypoxemic respiratory failure: a systematic review," Respiratory Medicine, vol. 121, pp. 100-108, 2016.

[21] F. Stéphan, B. Barrucand, P. Petit et al., "High-flow nasal oxygen vs noninvasive positive airway pressure in hypoxemic patients after cardiothoracic surgery," JAMA, vol. 313, no. 23, pp. 2331-2339, 2015.

[22] G. Hernández, C. Vaquero, L. Colinas et al., "Effect of postextubation high-flow nasal cannula vs noninvasive 
ventilation on reintubation and postextubation respiratory failure in high-risk patients," JAMA, vol. 316 , no. 15, pp. 1565-1574, 2016.

[23] M. Okuda, N. Tanaka, K. Naito et al., "Evaluation by various methods of the physiological mechanism of a high-flow nasal cannula (HFNC) in healthy volunteers," BMJ Open Respiratory Research, vol. 4, no. 1, Article ID e000200, 2017.

[24] R. L. Parke, A. Bloch, and S. P. McGuinness, "Effect of veryhigh-flow nasal therapy on airway pressure and end-expiratory lung impedance in healthy volunteers," Respiratory Care, vol. 60 , no. 10, pp. 1397-1403, 2015.

[25] T. Mauri, C. Turrini, N. Eronia et al., "Physiologic effects of high-flow nasal cannula in acute hypoxemic respiratory failure," American Journal of Respiratory and Critical Care Medicine, vol. 195, no. 9, pp. 1207-1215, 2017.

[26] C. L. Su, L. L. Chiang, S.-H. Yang et al., "Preventive use of noninvasive ventilation after extubation: a prospective, multicenter randomized controlled trial," Respiratory Care, vol. 57, no. 2, pp. 204-210, 2011.

[27] J. P. Frat, A. W. Thille, A. Mercat et al., "High-flow oxygen through nasal cannula in acute hypoxemic respiratory failure," The New England Journal of Medicine, vol. 372, no. 23, pp. 2185-2196, 2015.

[28] T. N. Liesching and Y. Lei, "Efficacy of high-flow nasal cannula therapy in intensive care units," Journal of Intensive Care Medicine, vol. 34, 2017.

[29] H.-W. Huang, X.-M. Sun, Z.-H. Shi et al., "Effect of high-flow nasal cannula oxygen therapy versus conventional oxygen therapy and noninvasive ventilation on reintubation rate in adult patients after extubation: a systematic review and metaanalysis of randomized controlled trials," Journal of Intensive Care Medicine, vol. 33, no. 11, pp. 609-623, 2018.

[30] A. Gray, S. Goodacre, D. E. Newby, M. Masson, F. Sampson, and J. Nicholl, "Noninvasive ventilation in acute cardiogenic pulmonary edema," New England Journal of Medicine, vol. 359, no. 2, pp. 142-151, 2008.

[31] F. M. Vital, M. T. Ladeira, and A. N. Atallah, "Non-invasive positive pressure ventilation (CPAP or bilevel NPPV) for cardiogenic pulmonary edema," The Cochrane Database of Systematic Reviews, vol. 31, no. 5, Article ID CD005351, 2013.

[32] N. Schwabbauer, B. Berg, G. Blumenstock, M. Haap, J. Hetzel, and R. Riessen, "Nasal high-flow oxygen therapy in patients with hypoxic respiratory failure: effect on functional and subjective respiratory parameters compared to conventional oxygen therapy and non-invasive ventilation (NIV)," $B M C$ Anesthesiology, vol. 14, p. 66, 2014.

[33] A. W. Thille, F. Boissier, H. Ben-Ghezala et al., "Easily identified at-risk patients for extubation failure may benefit from noninvasive ventilation: a prospective before-after study," Critical Care, vol. 20, p. 48, 2016.

[34] B. Rochwerg, L. Brochard, M. W. Elliott et al., "Official ERS/ ATS clinical practice guidelines: noninvasive ventilation for acute respiratory failure," European Respiratory Journal, vol. 50, no. 2, Article ID 1602426, 2017. 